\title{
Reviewers 2002 (volume 86)
}

The editors are grateful to the following, who assisted in the assessment of papers during the past year.

\begin{tabular}{|c|c|c|c|}
\hline Abramson David H & Boulton Mike & Curcio Christine & Flynn John T \\
\hline Acheson James F & Bourke Robert D & Damato BE & Folberg Robert \\
\hline Adamis Anthony $\mathrm{P}$ & Bowd Christopher J & Damji Karim F & Forrester John V \\
\hline Ainsworth John & Bradford, Jr Reagan H & Dana Reza & Forster David J \\
\hline Aisenbrey Sabine & Brandt James & Danis Ronald Peter & Foster Allen \\
\hline Akama Tomoya & Branley Michael & Dart John & Foster Bradley S \\
\hline Akduman Levent & Brinton Gregory S & Davey Clare & Foster David \\
\hline Alcorn Deborah & Brodsky Michael & Dawson William W & Foster Paul \\
\hline Ali Robin & Bron AJ & Daya Sheraz M & Fournier AV \\
\hline Alio Jorge & Brooks A & De Kozak Yvonne & Fox Gregory M \\
\hline Allen David & Brooks, Jr H Logan & De Potter Patrick & Fraunfelder F \\
\hline Alm Albert & Brown Melissa M & de Smet M & Frederick Douglas \\
\hline Alpar John & Buckley Roger & Dees C & Freeman William R \\
\hline Alsbirk Poul & Bunce Catey & Demirci Hakan & Friberg Thomas R \\
\hline Alward Wallace & Burk Scott E & Desai Parul & Friedberg Mark A \\
\hline Anderson Douglas & Burke Miles J & Dev Sundeep & Friedlaender Mitchell H \\
\hline Anderson Richard L & Burnstine Michael A & Dhaliwal Deepinder K & Friedman Alan $\mathrm{H}$ \\
\hline Araie Makoto & Calonge Margarita & Dhar-Munshi Sushma & Frost NA \\
\hline Arden Geoffry & Campos Emilio & Diamond Jeremy & Frucht-Pery Joseph A \\
\hline Arend Oliver & Cantor Louis & Dick Andrew & Fujii Gildo Y \\
\hline Armbrecht Ana M & Cantore William & Dickinson CM & Fujikado Takashi \\
\hline Armitage John & Carelli Valerio & Digre Kathleen & Gallagher Patrick \\
\hline Armitage WJ & Carney Leo & Donahue Sean P & Gallemore Ron P \\
\hline Arnold Jennifer & Carter Keith D & Donnenfeld Eric D & Gallie Brenda \\
\hline Arroyo Jorge G & Caspers-Velu L & Douglas Gordon R & Gandolfi S \\
\hline Astbury Nick & Casson Evanne J & Downing John E & Gans Laurence A \\
\hline Atebara Neal H & Cavanagh H Dwight & Dresner Steven C & Garweg Justus \\
\hline Atta HR & Chakravarthy U & Drewry, Jr Richard D & Gaudio Paul Anton \\
\hline Austin Mike & Chan Chi-Chao & Driver Paul J & Gavin MP \\
\hline Aylward Bill & Chang David & Dua HS & Geiger Greel L \\
\hline Ayyala Ramesh S & Chang Tom S & Dubiner Harvey B & Gelwan Mark J \\
\hline Azuara-Blanco Augusto & Char Devron & Dugel Pravin U & Gentile Ronald C \\
\hline Azzarolo Anna Maria & Charles Stephen & Duncan George & Gentle Alex \\
\hline Bachynski Brian N & Chaum Edward & Duncan Jacque & Giblin Frank J \\
\hline Bailey Clare & Chisholm I & Dunlop Anthony A & Gibson Jonathan \\
\hline Baker John D & Chodosh James & Dunn, Jr James Philip & Gilbard Jeffrey P \\
\hline Ball Karlene & Chou Tina M & Dyer Michael & Gilbert Claire \\
\hline Barbazetto Irene A & Chua John & Egbert Peter R & Gillies Mark C \\
\hline Barr Charles C & Chumbley Lee C & Eiferman Richard A & Gitter Kurt A \\
\hline Bartley George B & Churchill A & El-Ashry Mohamed & Goldberg Ivan \\
\hline Bateman J Bronwyn & Ciulla Thomas A & Elder Mark J & Goldberg Robert \\
\hline Bates Adrian K & Claridge KG & Eliott Dean & Golnick Karl C \\
\hline Battegay E & Clark Abbot & Eller Andrew W & Gombos Dan S \\
\hline Baudouin Christophe & Clarke Michael P & Ellis John & Gonnering Russell S \\
\hline Bauer Dirk & Cleary Marie & Elston JS & Good Margaret S \\
\hline Baum Jules & Clement Richard & Ernest J Terry & Good William V \\
\hline Baumal Caroline & Clinch Thomas E & Escobar-Gomez Marcela & Gottlieb Justin \\
\hline Beauchamp George & Cole Mike & Essock Edward & Gottlob Irene \\
\hline Beaumont Paul & Coleman Anne & Eustis, Jr H Sprague & Gottsch John D \\
\hline Becker Bruce B & Collin JRO & Evans Tim & Gragoudas Evangelos S \\
\hline Beckman Kenneth A & Conway Mandi D & Ezra Eric & Graham E \\
\hline Bejjani Bassem & Conway Stephen T & Fechtner Robert D & Gray Roger \\
\hline Belkin Michael & Corbett James & Feder Robert S & Greaney Michael J \\
\hline Bennett HGB & Corbett Melanie C & Feke Gilbert & Greenberg Paul \\
\hline Bhisitkul Robert & Cordeiro Francesca & Fekrat Sharon & Gregory-Evans Kevin \\
\hline Bird Alan & Cordeiro Maria & Ferguson Veronica & Gregson R \\
\hline Bishop Paul & Coscas Gabriel J & Ferris Frederick & Greiner Jack Volker \\
\hline Black G & Costa Vital & Fielder Alistair & Grewal Sanjeev \\
\hline Blackman H Jane & Coster DJ & Figueiredo Francisco & Grierson I \\
\hline Bloom P & Cottriall Charles & Finger Paul T & Grizzard W Sanderson \\
\hline Blumenthal Eytan Z & Courtright Paul & Fiscella Richard G & Gross Jeffrey G \\
\hline Boergen Klaus-Peter & Cowen David E & Fishman Gerald A & Grossniklaus Hans \\
\hline Boldt HC & Crafoord Sven & Fitzke FW & Gupta Lopa Y \\
\hline Bolling James P & Cree Ian & Fitzpatrick D & Guthoff Rudolf F \\
\hline Bonnano Joseph & Cristol Stephen & Flach Alan & Haites N \\
\hline Booton Gregory & Criswell Mark & Flaxel Christina J & Hales Angela M \\
\hline Borderie Vincent & Cruz Oscar Alfredo & Fleck Brian & Halperin Lawrence S \\
\hline Born Christopher & Cunningham, Jr Emmett T & Flynn Harry W & Hamilton Andrew \\
\hline
\end{tabular}


Han Dennis P

Handa James T

Harbour J William

Hardcastle Alison J

Harding SP

Hardten David

Harper Robert

Harrad Richard

Harris, Jr David J

Harrison Andrew R

Harweth Ronald S

Haynes RJ

Hedges, III Thomas R

Heier Jeffrey $\mathrm{S}$

Held Richard M

Henson DB

Heon Elise

Herbort Carl

Hettinger Michael E

Heyderman Rob

Hidayat Ahmed A

Hill John

Hill Richard A

Hirakata Akito

Hiscott

Hitchings Roger A

Hitzenberger Christopher

Ho Chi Kin

Hoh Sek-Tien

Holder Graham

Holds John

Holland Gary

Holz Eric

Horowitz Amy

Horton Jonathan

Howland Howard

Hoyt Creig S

Hsuan James

Huang Andrew J

Huang David

Hundal KS

Hungerford J

Hykin Philip

Ie Darmakusuma

Iester Michele M

Inglehearn Chris

Inglehearn Christopher

Irvine Alexander

Ishikawa Hiroshi

Jacobson Daniel

Jager Martine

Jampel Henry D

Jay JL

Jay Walter M

Johnson Chris A

Johnson Douglas H

Johnson G

Johnson Lenworth

Johnson RW

Jonas Jost B

Jonasson Fridbert

Jones NP

Joyce Nancy

Jumper Michael J

Kador Peter F

Kakehashi Akihiro

Kalenak Jeffrey Ward

Kanski Jack J

Kao Winston

Kaplan Joseline

Karickhoff John R

Kashii Satoshi

Katowitz James A

Katz Barrett

Katz Douglas G

Katz Harold R

Katz Joanne

Kaufman Herbert
Kaye Laurence D

Keating David

Keech Ronald V

Keeffe Jill

Keegan David

Keltner John I

Kemp Ewan

Kennerdell John S

Kenyon Kenneth R

Kergoat Helene

Keunen Jan

Khan Jemshed A

Khan Lim Doreen

Khaw Peng

Khawly Joseph A

Khouri Albert S

Kijlstra Aize

Kilmartin Dara

Kirchhof Bernd

Kirkness Colin

Kirkpatrick JNP

Kleiner Robert C

Klyce Stephen

Knepper Paul A

Kobayashi Hiroshi

Kokame Gregg T

Kook Michael S

Kooner Karanjit

Kornstein Howard S

Krishna Rohit

Krupin Theodore

Kuhn Ferenc

Kuo Irene $\mathrm{C}$

Kupersmith Mark J

Kushner Burton

Kwok Alvin

Lachkar Yves

Lahey J

Laibson Peter

Lam Byron L

Lambert Scott

Lamberts David W

Landau Klara

Larkin DFP

Larkin G

Latina Mark A

Lauer Simeon A

Lavin Mike

Lavin MJ

Leatherbarrow B

Lee Andrew G

Lee Graham

Lee JP

Lehmann Ordan

Lehoang Phuc

Lemp Michael A

Leonardi Andrea

Leske M Christina

Lesser Robert L

Leuck CJ

Levin Leonard

Lewis Geoffrey

Li Helen Ka-Fun

Lietman T

Lightman Susan

Lim Jennifer Irene

Lin Robert T

Lin Shan

Linberg John V

Lipshitz Isaac

Little BC

Liu Christopher S

Liu Don

Lloyd Mary Ann

Lobes, Jr Louis A

Lois Noemi

Lotery Andrew

Lovicu Frank J
Lowder Careen

Lowenstein Anat

Luck Jonathan

Luthert P

Luttrull Jeffrey

Lutz Jean-Michel

Ma David

Maccumber Matthew W

Macdonald Ian M

MacEwen Carrie

Mackey David

Mader Thomas $\mathrm{H}$

Madreperla Steven A

Maeda Naoyuki

Magargal Larry E

Majid Mo

Malinowski Susan

Mamalis Nick

Manners R

Marfurt Carl F

Margo Curtis E

Margolis Todd

Markham A

Markham RHC

Marmor Michael

Marquart Mary E

Marshall John

Martin Frank Joseph

Masi Robert J

Masket Samuel

Massin Pascale G

Massof Robert

Matsuo Toshihiko

Mattox Cynthia

Mawn Louise A

Mayer Eric

McAvoy John W

McCannel Colin

McCartney David L

McCarty Catherine A

McCluskey P

McDonnell Peter J

McFadzean RM

McGhee Charles

McIlwaine $\mathrm{G}$

McKechnie Nicol

McKendrick Allison

McLeod David

McLeod S

McMullan Frances Dickinson

McNaught AI

Mein Calvin E

Menage M

Merrill Pauline T

Mets Marilyn B

Meyer Eric

Michon John

Mieler William F

Mifflin Mark D

Miller Darlene

Miller Neil R

Minassian DC

Mindel Joel S

Mintz-Hittner Helen

Miyake Kensaku

Miyamoto Kazuaki

Mo Jun

Mojon Daniel

Molteno Anthony CB

Moore AT

Moore T

Moorthy Ramana S

Morgan JE

Morris Robert E

Morse Lawrence S

Moseley $M$

Moshfeghi Darius

Moss Scot

Muchnick Richard S

Mumford David

Munoz Beatriz E

Murphy Robert P

Murray Phil

Musco Paul Sebastian

Mutti Donald

Naumann Gottfried O

Nelson Duane J

Nerad Jeffrey A

Neumann Ron

Newbury-Ecob R

Newman Nancy

Newman Steven

Nicolela Marcelo

Nishi Okihiro

Noble David

O'Brart David

O'Brien Colm

$\mathrm{O}^{\prime}$ Brien Terrence

O'Callaghan Richard J

O'Day Justin

Odom J Vernon

Okumura Atsushi

Olk R Joseph

Olson John

Olver Jane M

Ormerod Ian

Owsley Cynthia

Palmer James D

Palmer Steven S

Pandey Suresh K

Parks Stuart

Parrish, II Richard K

Pasquale Louis

Paul T Otis

Pavesio Carlos

Pavlin Charles J

Penfold Philip

Pennington TH

Perry Arthur C

Perry Henry D

Peters Tim

Peyman Gholam A

Pierce Eric

Pierce William M

Pirnaza Ramin

Pleyer U

Poinoosawmy D

Pollock Stephen C

Poon Alex

Potash Seth David

Potts M

Price Francis W

Price N

Pulido Jose S

Purvin Valerie 
Ritch Robert

Robb Richard

Roberts Anita

Roberts-Harry J

Robin Alan

Robin Todd

Robinson K

Rockwood Edward J

Rootman David

Rootman Jack

Rosa Robert

Rosen E

Rosenbaum James T

Rosenwasser George

Rosner Mordechai

Rothova A

Roy Monique S

Roy Sayon

Rubin Gary

Rubsamen Patrick E

Ruderman Jon M

Ruether Klaus

Russell Paul

Russell-Eggitt I

Ryan, Jr Edwin Hurlbutt

Sadun Alfredo A

Salmon John F

Sample Pamela A

Samples John R

Sandberg Michael

Sanders Donald R

Savino Peter J

Saw Seang-Mei

Scanlon Peter

Schachat Andrew

Schatz Martha P

Schechter Barry A

Schmetterer Leopold

Schmidt-Erfurth Ursula M

Schuman Joel

Schwab Ivan R

Schwartz Arthur L

Schwartz Steven D

Scott Ingrid U

Scott J Angus

Scott William

Sebag Jerry
Seitz Berthold

Sergott Robert C

Seward HC

Shah Gaurav

Shammas H John

Sharara Nariman

Sharp Peter

Shields Carol L

Shuttleworth Garry

Silvestri G

Sires Bryan $\mathrm{S}$

Skuta Gregory L

Slakter Jason

Slavin Michael L

Sloper J

Smit Barbara

Smith J

Smith S Gregory

Smith Valerie

Snibson Grant

Solley Wayne A

Sommer Alfred

Soto Noel Perez

Soubrane Gisele

Souza-Diaz Carlos

Spadea Leopoldo

Spaeth George

Spalding Samuel C

Spalton DJ

Spitznas Manfred

Spoor Thomas C

Spry Paul

Stamper Robert

Stanford M

Stanley John

Starck Tomy

Starr Michael B

Stechschulte $\mathrm{S}$

Steuer Eric R

Stewart William

Stjernschantz Johan W

Stone E

Streeten Barbara W

Sugar Aalan

Sugar Joel

Sumich PR

Sunaric-Megevand Gordana
Susanna Remo

Ta Christopher

Tabandeh Homayoun

Tabbara Khalid

Tabin Geoffrey

Tang Rosa A

Tasman William S

Taylor Hugh

Taylor John RW

Teitelbaum Charles S

Tello Celso

Tessler Howard

Tezel Tongalp

Thomas Edgar

Thompson John

Timmers Adrian

To Chi

Tole Derek

Tomsak Robert

Topouzis Fotis

Toris Carol B

Tornqvist Goran P

Tosini Gianluca G

Towler Hamish MA

Traboulsi Elias I

Trese Michael T

Troia Robert N

Trope Graham

Tseng Scheffer CG

Tso Mark

Tuli Sonal S

Twa Michael D

Tychsen Lawrence

Tyers AG

Tyers Tony

Vajpayee Rasik B

Vernon SA

Votruba Marcela

Vrabec Michael P

Wade N Kevin

Wallace GR

Walton David S

Walton R Christopher

Wang Jie

Warburg Mette

Webster Andrew

Weinberg David A
Weinreb Robert

Weissgold David

Weiter John J

Welsh Michael G

Werner Liliana

Wessels Izak F

West Sheila K

Whitcher Jack

White William L

Wiechmann Allan F

Wild JM

Wilhelmus Kirk R

Wilkinson CP

Williams Cathy

Williams Keryn

Williamson $\mathrm{TH}$

Willig Jeffrey L

Willshaw HE

Wilson M Roy

Wilson Matthew W

Wise James B

Wishart Peter

Wittpenn, Jr John R

Wong David

Wong Elaine

Wong Tien

Wong Tina

Wood Thomas O

Woog John J

Wormald $\mathrm{R}$

Wormstone Ian

Wright Kenneth W

WuDunn Darrell

Yamada Jun

Yamamoto Tetsuya

Yang YC

Yannuzzi Lawrence A

Yap Maurice

Yolton Robert L

Zabriskie Norm A

Zadnik Karla

Zakov Nicholas

Zamir Ehud

Zangwill Linda

Zarbin Marco

Zeimer Ran

Zierhut M

Zierhut Manfred

Zimmerman Neal J 